\title{
SKIL facilitates tumorigenesis and immune escape of NSCLC via upregulating TAZ/autophagy axis
}

\author{
Fang Ma', Meng-Ge Ding ${ }^{1}$, Yi-Yu Lei ${ }^{1}$, Li-Hua Luo², Shun Jiang ${ }^{1}$, Yu-Hua Feng ${ }^{1}$ and Xian-Ling Liu(i)
}

\begin{abstract}
Immune escape is an important mechanism in tumorigenesis. The aim of this study was to investigate roles of SKIL in tumorigenesis and immune escape of non-small-cell lung cancer (NSCLC). SKIL expression levels in NSCLC cell line, clinical sample, and adjacent normal tissue were measured by quantitative PCR, western blot, or immunohistochemistry. Lentivirus was used to overexpress/silence SKIL or TAZ expression. Malignant phenotypes of NSCLC cells were evaluated by colony formation, transwell, and MTT assays, and in xenograft mice model. Syngeneic mice model and flow cytometry were used to evaluate T cell infiltration. Quantitative PCR and western blot were applied to evaluate relevant mRNA and protein levels, respectively. Co-immunoprecipitation was applied to unveil the interaction between SKIL and TAZ. SKIL expression was higher in NSCLC tissue compared to adjacent normal tissue. Silencing of SKIL inhibited malignant phenotypes of NSCLC cells and promoted T cell infiltration. SKIL-knockdown inhibited autophagy and activated the STING pathway in NSCLC cells through down-regulation of TAZ. Silencing of TAZ cancelled the effects of SKIL overexpression on malignant phenotypes and autophagy of NSCLC cells. Inhibition of autophagy reversed the effects of SKIL/TAZ overexpression on the STING pathway. In conclusion, SKIL promoted tumorigenesis and immune escape of NSCLC cells through upregulation of TAZ/autophagy axis and inhibition on downstream STING pathway.
\end{abstract}

\section{Introduction}

Non-small-cell lung cancer (NSCLC) remains a major global health threat, which affects 1.8 million people and causes an estimated 1.59 million death worldwide ${ }^{1}$. Although currently there are several available treatments for NSCLC (e.g. surgery, radiotherapy, chemotherapy, and targeted therapy), the prognosis of NSCLC patients is still unsatisfactory ${ }^{2}$. In the past few years, immunotherapy has emerged as a new treatment method for cancer. Immune system plays important roles in eliminating cancer cells before they can form clinically identifiable tumor. In order to survive this immune pressure, some of the cancer cells develop a way to escape the surveillance of immune

\footnotetext{
Correspondence: Xian-Ling Liu (liuxianling@csu.edu.cn)

${ }^{1}$ Department of Oncology, The Second Xiangya Hospital, Central South

University, 410011 Changsha, Hunan Province, P.R. China

${ }^{2}$ Cancer Center, Union Hospital, Tongji Medical College, Huazhong University

of Science and Technology, 430022 Wuhan, P.R. China

These authors contributed equally: Fang Ma, Meng-Ge Ding

Edited by J.-E. Ricci
}

system (so-called immune escape), mainly through interacting with immune checkpoint molecules expressed on regulatory $\mathrm{T}$ cells (e.g. cytotoxic T-lymphocyte antigen-4 (CTLA-4) and programmed death-1 (PD-1)) and subsequent inhibition of the activation of regulatory $\mathrm{T}$ cells $\mathrm{s}^{3}$. Immunotherapy targeting those immune checkpoint molecules has been intensively studied and achieved success in clinical trials in NSCLC 4 .

Cyclic GMP-AMP (cGAMP) synthase (cGAS) and stimulator of interferon (IFN) gene (STING) were reported to play important roles in anti-cancer immunity, e.g. T cell immunity ${ }^{5-9}$. cGAS is a key cytosolic DNA sensor. When cytosolic DNA (e.g. microbial DNA) is detected, cGAS produces cGAMP which promotes the translocation of STING from endoplasmic reticulum and Golgi apparatus to perinuclear vesicles ${ }^{10,11}$. Re-located STING could activate TANK-binding kinase 1 (TBK1), NF- $\kappa B$, and interferon regulatory transcription factor 3 (IRF3), which then stimulate the production of IFN, an essential 
factor in anti-cancer immunity ${ }^{12}$, and other inflammatory cytokines ${ }^{10}$. Previous studies reported that inhibiting DNA damage response proteins (poly-ADP-ribose polymerase (PARP) and checkpoint kinase 1 (CHK1)) could activate cGAS-STING-mediated anti-cancer immunity, and enhance the blockade of PD-1 checkpoint and infiltration of cytotoxic $\mathrm{T}$ cell ${ }^{13,14}$. Those findings potentiate the cGAS-STING pathway as a therapeutic target to enhance the efficacy of PD-1 immune checkpoint inhibitors.

Firstly discovered as a proto-oncogene, SKIL (also known as SnoN) is a mediator of the transforming growth factor- $\beta$ (TGF- $\beta$ ) signaling pathway and exerts prooncogenic activity through inhibiting TGF- $\beta /$ Smad pathway $^{15}$. Overexpression of SKIL was reported in esophageal squamous cell carcinoma (ESCC) ${ }^{16}$ and breast cancer ${ }^{17}$, and amplification of SKIL was also found in NSCLC, prostate cancer, and head and neck squamous carcinoma $(\mathrm{HNSC})^{18}$. In addition, SKIL was involved in reduction of autophagy and subsequent tumor cell apoptosis in arsenic trioxide-treated promyelocytic leukemia ${ }^{19}$ and ovarian carcinoma $^{20}$. Evidence also showed that expression of SKIL was elevated in BCL11A-overexpressed lung squamous carcinoma ${ }^{21}$. On the other hand, some studies suggested suppressive roles of SKIL on the proliferation and survival in some specific pathological subtypes of cancer (e.g. lower grade esophageal adenocarcinoma, lower grade ovarian adenocarcinoma, pancreatic adenocarcinoma, and breast ductal adenocarcinoma ${ }^{22}$ ), and silencing of SKIL (or SnoN) upregulated proliferation of ESCC cell line ${ }^{23}$, indicating a complex of SKIL in tumorigenesis. In addition, no study has reported role of SKIL in immune escape.

YAP/TAZ is closely-related transcriptional regulators and plays important roles in many biological processes, e.g. organ growth, tissue biology, and cancer ${ }^{24,25}$. In addition, activation of YAP/TAZ was found in several types of cancer and associated with cancer proliferation, progression, and metastasis ${ }^{26,27}$. YAP/TAZ receives input from several signaling pathways, including Hippo pathway, Wnt pathway, and $\mathrm{F}$-actin ${ }^{24}$. For example, as important effector of the Hippo pathway, YAP/TAZ could be phosphorylated and inhibited by key members of Hippo complex, Lats2 and Salvador $(\mathrm{Sav})^{28,29}$. On the other hand, YAP/TAZ regulates many downstream signaling factors, e.g. connective tissue growth factor (CTGF), cysteine-rich angiogenic inducer 61 (CYR61), and modulates interactions between cells and extracellular matrix ${ }^{30}$. Similar to SKIL ${ }^{19,20}$, YAP/TAZ was also reported to assist cancer cells to bypass the normal contact inhibition by promoting autophagy through regulation on expression of myosin-II and F-actin stress fibers ${ }^{31}$, which pathway is commonly compromised in normal noncancerous cells. Interestingly, a recent study reported that SKIL promoted the tumorigenesis of breast cancer by enhancing the activity of $\mathrm{TAZ}^{32}$, which might be an important mechanism for the oncogenic effects of SKIL.

In our study, we investigated the relationship between SKIL and TAZ, and their functions on autophagy and malignant phenotype of NSCLC. We found that SKIL induced malignant phenotypes of NSCLC through upregulation of TAZ pathway and autophagy, which also led to inhibition on immune escape from $\mathrm{T}$ cell immunity through inhibition on the STING pathway.

\section{Methods}

\section{Sample collection and ethics}

Human NSCLC and paired adjacent normal tissue samples were collected from patients who received surgery from 2017 to 2019 in Department of Oncology, The Second Xiangya Hospital, Central South University. After removal of tumor, blocks of tumor tissue $(1 \mathrm{~cm} \times 1 \mathrm{~cm} \times 0.2 \mathrm{~cm})$ and adjacent normal tissue were collected and put into formalin-fixed and paraffin-embedded (FFPE) blocks following standard procedure in pathology department, or frozen at $-80^{\circ} \mathrm{C}$ for future analysis. Informed consent was obtained from each patient enrolled in our study. Our study protocol was approved by Institutional Ethics Committee of Central South University.

\section{Cell culture}

Human lung adenocarcinoma cell lines (HCC827, CALU-3, NCI-H1975), human lung carcinoma cell line (A549), human lung squamous cell carcinoma cell lines (NCI-H226, NCI-H520, SK-MES-1), human bronchial epithelial cell line (16HBE) which was immortalized from normal bronchial epithelial cells and retains their characteristic features, and anaplastic murine lung carcinoma cell line (Madison 109 lung carcinoma, M109) were obtained from Fenghui Biology (Hunan, China). All the cell lines have passed the STR authentication. After thawing, cell lines were cultured in RPMI-1640 culture medium supplemented with $10 \%$ fetal bovine serum and $1 \%$ penicillin/streptomycin. Cells were kept in humid atmosphere with $5 \%$ carbon dioxide at $37^{\circ} \mathrm{C}$. Culture medium was changed every other day and cells were subcultured when reached $80 \%$ confluence. All the cultured cells were routinely checked for mycoplasma contamination.

\section{Animal model}

To evaluate the in vivo tumorigenesis of cell lines, BALB/c-nu nude mice were obtained from Department of Laboratory Animals, Central South University, divided randomly into two groups, injected subcutaneously with $0.5 \times 10^{6}$ of tumor cells each. Size of tumor was monitored every 4 days using a caliper. Tumor volume was calculated by (tumor length $\times$ tumor width $\left.^{2}\right) / 2$. On day 24 , mice 
were euthanized by carbon dioxide and tumor blocks were collected from the animals for subsequent analysis. For syngeneic mice model, mouse lung cancer cell line (M109) and BALB/c mice were used instead, and $0.5 \times 10^{6}$ of cultured M109 cells were injected subcutaneously into each BALB/c mouse with Matrigel (1:1; BD Biosciences, USA). On day 21 , mice were euthanized by carbon dioxide and tumor blocks were collected. All procedures were approved by Department of laboratory animals, Central South University. The investigators were blinded to the group allocation during the experiment and when assessing the outcome.

\section{Flow cytometry}

The collected tumor blocks were firstly minced into pieces and single-cell suspension were obtained by passing the tumor mince through a cell strainer (Corning, USA). After fixation and permeabilization using BD Cytofix/Cytoperm kit (554714, BD Biosciences), cells were stained with anti-CD3 (100246, Biolegend), anti-CD4 (100526, Biolegend), anti-CD45 (101917, Biolegend), or anti-CD8 (100722, Biolegend) antibody following standard flow cytometry protocol. Results were then obtained using Fortessa platform (BD Biosciences) and analyzed using FlowJo software (BD Biosciences). CD3 and CD45 were used to analyze total T cells (both CD45 and CD3 positive). Cytotoxic $\mathrm{T}$ cells were further analyzed using CD4 and CD8 after gating of $\mathrm{CD}^{+}$cells, while cells from inguinal lymph node were used as reference of gating.

\section{Western blot}

Tissue or cell samples were homogenized in $1 \times$ phosphate-buffered saline (PBS). Protein concentration of the samples was determined using Pierce Rapid Gold BCA Protein Assay Kit (Themo Fisher, USA). One microgram of protein from each sample was loaded onto SDS-PAGE gel. After separation of the proteins using electrophoresis and transferring onto nitrocellulose membranes, targeted proteins were blocked in $5 \%(\mathrm{w} / \mathrm{v})$ skimmed milk in TBST. Proteins were then stained at $4{ }^{\circ} \mathrm{C}$ overnight with specific primary antibody: SNAIL1 (\#3895, CST), SLUG (\#9585, CST), E-cadherin (\#14472, CST), vimentin (\#3932, CST), GAPDH (\#97166, CST), STING (\#13647, CST), TBK1 (\#3504, CST), p-TBK1 (\#5483, CST), IRF3 (\#4302, CST), p-IRF3 (\#29047, CST), $\beta$-actin (\#58169, CST), Beclin-1 (\#3738, CST), p62 (\#39749, CST), LC3-I (\#4599, CST), LC3-II (\#2775, CST), TAZ (\#4883, CST), CTGF (\#10095, CST), CYR61 (\#39382, CST), LATS2 (\#5888, CST), Sav (\#13301, CST), or p-TAZ (\#59971, CST). On the next day, membranes were washed in $1 \times$ PBS, and cultured with anti-mouse IgG (\#7076, CST) or anti-rabbit IgG (\#7074, CST) secondary antibody depending on the type of primary antibody for $2 \mathrm{~h}$ at room temperature. Results were then visualized using gel- imaging system (GS-800, Bio-Rad, USA), followed by densitometric analysis.

\section{Quantitative PCR}

Total RNA was extracted using TRIzol reagents (Invitrogen, USA) following the manufacturer's instruction. Frozen tissue samples were thawed at room temperature. After thawing, $50-100 \mathrm{mg}$ tissue sample was homogenized in $1 \mathrm{ml}$ of TRIzol reagent. For cell samples, $1 \times 10^{6}$ cells were lysed in $1 \mathrm{ml}$ TRIzol reagent. After adding $0.2 \mathrm{ml}$ chloroform, sample homogenate was incubated for $2 \mathrm{~min}$ and centrifuged for $15 \mathrm{~min}$ at $12,000 \times g, 4{ }^{\circ} \mathrm{C}$. The upper aqueous phase was transferred into a new tube and mixed with equal volume of $70 \%$ ethanol. The samples were filtered into a spin cartridge by centrifugation and the flowthrough was discarded. The RNAs were then eluted using RNase-free water. After determination of RNA concentration by measuring absorbance of diluted samples at 260 and $280 \mathrm{~nm}$ using a spectrophotometer (NanoDrop, Thermo Scientific, USA), $1 \mu \mathrm{g}$ RNA from each sample was used for reverse transcription. Quantitative PCR (qPCR) was performed using SYBR Select Master Mix (Invitrogen, USA) on ABI 7500 system (ABI, USA) following a standard procedure. Specific primers were purchased from Sangon Biotech (Shanghai, China). Detailed sequences for each primer pair are: human CXCL10, sense GTGGCATTCAAGGAGTAC CTC, antisense TGATGGCCTTCGATTCTGGATT; hu man CCL5, sense CCAGCAGTCGTCTTTGTCAC, antisense CTCTGGGTTGGCACACACTT; human IFN- $\beta$, sense CATTACCTGAAGGCCAAGGA, antisense CAATT GTCCAGTCCCAGAGG; mouse CXCL10, sense CCCA CGTGTTGAGATCATTG, antisense GTGTGTGCGTG GCTTCACT; mouse CCL5, sense ATATGGCTCGGACA CCACTC, antisense TCCTTCGAGTGACAAACACG; SOX2, sense CCCACCTACAGCATGTCCTACTC, antisense TGGAGTGGGAGGAAGAGGTAAC; SKIL, sense A CCAGTTATTATTCCCCTGTTCCT, antisense GGCATG GCTTACCAGAAACC; CD44, sense CCAGAAGGAAC AGTGGTTTGGC, antisense ACTGTCCTCTGGGCTT GGTGTT; OCT3/4, sense CCTGAAGCAGAAGAGGAT CACC, antisense AAAGCGGCAGATGGTCGTTTGG; CD133, sense CACTACCAAGGACAAGGCGTTC, antisense CAACGCCTCTTTGGTCTCCTTG; NANOG, sense CTCCAACATCCTGAACCTCAGC, antisense CGTCA CACCATTGCTATTCTTCG.

\section{Transfection}

For overexpression of SKIL and TAZ genes, full coding region of target gene (human SKIL, mouse SKIL, human $T A Z$, or mouse $T A Z$ ) was amplified and cloned into lentivirus vector pLVX-IRES-Neo. For knockdown of SKIL and $T A Z$ genes, short hairpin RNA (shRNA) was purchased (Sangon Biotech, China) and cloned into pLVXIRES-Neo. Empty pLVX-IRES-Neo vector was used as 
control. The lentivirus vectors were then used for the transfection of target cells. The transfection was performed using Lipofectamine 2000 system (Thermo Fisher, USA) following the manufacturer's instruction. Cells were seeded in a six-well plate with packaging medium at $70-80 \%$ confluence and allowed to incubate overnight at $37^{\circ} \mathrm{C}$ in humidified atmosphere with $5 \% \mathrm{CO}_{2}$. On the next day, cells were transfected with lentivirus vectors and incubated at $37^{\circ} \mathrm{C}$ in humidified atmosphere with $5 \%$ $\mathrm{CO}_{2}$. Packaging medium was carefully replaced $6 \mathrm{~h}$ after the transfection. Forty-eight hours after the transfection, cells with stable transfection were selected using culture medium containing $1.5 \mu \mathrm{g} / \mathrm{ml}$ puromycin (Sigma-Aldrich, USA). After selection, culture medium was changed and cells with stable transfection were used for subsequent treatment and analysis.

\section{MTT assay}

3-(4,5-Dimethylthiazol-2-yl)-2,5-diphenyltetrazolium bromide (MTT) assay was performed to evaluate the viability of cells. Briefly, after suspension in culture medium, cultured cells were mixed with equal volume of $5 \mathrm{mg} / \mathrm{ml} \mathrm{MTT} \mathrm{(M2128,} \mathrm{Sigma,} \mathrm{dissolved} \mathrm{in} 1 \times$ PBS) and incubated at $37^{\circ} \mathrm{C}$ for $1 \mathrm{~h}$. After removing medium, $200 \mu \mathrm{l}$ DMSO was used to suspend MTT metabolic product. Mixture was incubated at $37^{\circ} \mathrm{C}$ for $10 \mathrm{~min}$, and optical density (OD) was measured at $490 \mathrm{~nm}$.

\section{Colony formation assay}

Briefly, cultured cells were trypsinzed and suspended in culture medium. Four thousand cells were then suspended in culture medium containing $0.4 \%$ low-meltingpoint agarose (Sigma, USA), which was overlaid on hardened $1.2 \%$ agarose bottom layer in $60 \mathrm{~mm}$ dishes. After cooling, the dishes were incubated at $37^{\circ} \mathrm{C}$ in humidified atmosphere with $5 \% \mathrm{CO}_{2}$. Culture medium was changed every 3 days. On day 14, colonies were stained with $1 \%$ crystal violet, and number of colonies which were larger than $200 \mu \mathrm{m}$ was counted under a light microscope (Leica Microsystems, USA) and recorded.

\section{Transwell assay}

Transwell assay was performed to evaluate the migration and invasion ability of cells. Transwell inserts suitable for 24-well plates $(8.0 \mu \mathrm{m}$ pores, Corning, USA) were used. For cell invasion ability analysis, the down side of the transwell membrane on the inserts was coated with Matrigel (Corning, USA, diluted in cold DMEM) at $4{ }^{\circ} \mathrm{C}$, and incubated at $37^{\circ} \mathrm{C}$ for $30 \mathrm{~min}$ to allow gelling. After reaching $50-60 \%$ confluence, culture cells were trypsinized and suspended in culture medium. Cell suspension was placed into upper chamber of the insert with Matrigel, and the insert was put into a sterile 24-well plate containing $500 \mu \mathrm{l}$ culture medium in each well. For cell migration ability analysis, the re-suspended cells were placed in to upper chamber without Matrigel. After incubation for $24 \mathrm{~h}$ in humidified atmosphere with $5 \%$ $\mathrm{CO}_{2}$, cells on the upper side of the insert membrane was completely removed using cotton swab. Inserts were fixed using $4 \%$ polyfluoroalkoxy and stained with $1 \%$ crystal violet. Invasion or migration ability of cells was evaluated by number of cells attached to the lower side of the insert. Quantification of the cells was performed by imaging of the insert membranes and subsequent analysis using ImageJ.

\section{Co-immunoprecipitation}

Immunoprecipitation was performed according to Zhu et al. $^{32}$. Briefly, high-salt lysis buffer was prepared using $420 \mathrm{mM} \mathrm{NaCl}, 50 \mathrm{mM}$ HEPES-KOH (pH 7.8), $5 \mathrm{mM}$ EDTA, 0.1\% NP-40, $3 \mathrm{mM}$ dithiothreitol (Sigma-Aldrich, USA), $0.5 \mathrm{mM}$ PMSF (Sigma-Aldrich, USA), and $10 \mu \mathrm{g} / \mathrm{ml}$ aprotinin (Sigma-Aldrich, USA). Cells were lysed in highsalt lysis buffer. Before the immunoprecipitation, cell lysates were cleared using protein A-Sepharose beads (Proteintech, IL, USA). Protein A-Sepharose beads coupled with anti-SKIL antibody (19218-1-AP, Proteintech, IL, USA) were then used to precipitate endogenous SKIL in cell lysates. Precipitated proteins were subject to further western blot analysis.

\section{Immunofluoresence staining}

Cells were firstly let grow on coverslips. Before staining, cells were fixed in $4 \%$ formaldehyde in $10 \%$ methanol for 20 min. After blocking with $10 \%$ goat serum, cells were cultured with diluted LC3B primary antibody (\#12741, CST, USA) for $1 \mathrm{~h}$, and then secondary goat anti-rabbit IgG antibody conjugated with Alexa Fluor 594 (\#8889, CST, USA) for $1 \mathrm{~h}$ at room temperature. After washing with $1 \times$ PBS, cells were stained with ProLong Gold Antifade Mountant with DAPI (P36935, Themo Fisher, USA). Result was quickly visualized under a fluorescence microscope (Leica Microsystems, USA) with excitation at $561 \mathrm{~nm}$.

\section{Immunohistochemistry}

Tumor and normal tissue were fixed in formalin for $24 \mathrm{~h}$, and then dehydrated and embedded in paraffin following standard pathology procedure in pathology department. The FFPE blocks were then sectioned at $4 \mu \mathrm{m}$ and stored at $4{ }^{\circ} \mathrm{C}$. On the day of staining, slides were deparaffinized in xylene followed by ethanol, and then hydrated in de-ionized water. For antigen-retrieval, slides were heated in Tris-EDTA buffer ( $\mathrm{pH}$ 9.0) in a pressure cooker for $3 \mathrm{~min}$ at full pressure and allowed to cool down in running tap water for $10 \mathrm{~min}$. After washing with $1 \times$ PBS, slides were stained with anti-SKIL primary antibody (1:50, ab78979, abcam, USA) for $1 \mathrm{~h}$, and then 
HRP-conjugated anti-rabbit secondary antibody (1:1000, ab6721, Abcam, USA). Staining result was visualized using 3,3'-diaminobenzidine (DAB, D8001, SigmaAldrich, USA) under a light microscope (Leica Microsystems, USA).

\section{Statistical analysis}

Statistical analysis was performed using GraphPad Prism 7.0. Data meet normal distribution. Variance was similar between the groups that are being statistically compared. Student's $t$-test was used to compare between two groups, while one-way ANOVA were used for multiple group comparison. $P<0.05$ was considered statistically significant.

\section{Results}

SKIL and TAZ expression levels are higher in NSCLC tissue

In all, we collected tumor and adjacent tissue samples from 32 NSCLC patients, of which 15 patients had lung adenocarcinoma (LUAD) and 17 had squamous carcinoma (LUSC). Measurement of SKIL expression using qPCR and IHC showed that SKIL expression was significantly higher in NSCLC tissue (Fig. 1a, f). Analysis using western blot showed increased SKIL protein expression in NSCLC tumor tissue compared to adjacent normal tissue, and a positive correlation was found between SKIL mRNA and protein expression levels in tumor (Fig. S1A, B). Further subgroup analysis showed that SKIL expression was significantly higher in LUSC and LUAD tissue compared to adjacent normal tissue $(P<$ 0.05 , see Fig. 1b, c). We also investigated SKIL expression of NSCLC samples using TCGA database, and obtained similar results in LUSC (Fig. 1e) but not in LUAD (Fig. 1d). Screening of SKIL expression levels in lung cancer cell lines showed that SKIL mRNA expression was elevated in some of LUAD cell lines (NCI-H1975 and CALU-3) and LUSC cell lines (NCI-H520 and NCI$\mathrm{H} 226)$, compared to normal lung cell line (16HBE), while SKIL level was not elevated in HCC827, A549, and SKMES-1 cell lines (Fig. 1g). Western blot results showed that SKIL protein expression was elevated in HCC827, A549, NCI-H1975, CALU-3, NCI-H520, and NCI-H226 cell lines, compared to control. Correlation analysis also showed a positive correlation between SKIL mRNA and protein expression levels in human lung cancer cell lines (Fig. S1C, D). Measurement of TAZ expression using qPCR also revealed increased TAZ mRNA levels in NSCLC tumor tissue compared to adjacent normal tissue (Fig. 1h). Correlation analysis showed a positive correlation between the relative SKIL expression levels and TAZ expression levels in tumor tissue of NSCLC patients (Fig. 1i). Results of lung cancer cell lines showed significantly higher TAZ expression levels in NCI-H226, NCI-H520, SK-MES-1, NCI-H1975, and HCC827 compared to control (16HBE), which was not seen in CALU-3 and A549 cell lines (Fig. 1j). This trend was different from the observations in SKIL expression in lung cancer cell lines (Fig. 1g). Overall, those results indicate that SKIL and TAZ expression levels are elevated in NSCLC. Based on the screening results from cell lines, CALU-3 (LUAD) and NCI-H520 (LUSC) which showed high expression of SKIL were chosen for subsequent analysis.

\section{Silencing on SKIL expression inhibited malignant phenotype and in vivo growth of CALU-3 and NCI-H520}

In order to understand the relationship between SKIL and malignant phenotype of NSCLC, we silenced SKIL in CALU3 and NCI-H520. Based on qPCR results, we selected shSKIL\#4 for subsequent analysis, which showed the most significant knockdown on SKIL expression in both cell lines (Fig. 2a). In addition, complementation experiment using another shRNA (shSKIL\#2) showed that SKIL overexpression could reverse the effects of shSKIL\#2 on cell viability, migration, invasion, and epithelial-mesenchymal transition (EMT) of NCI-H520 cell line (Fig. S3).

After SKIL was silenced by the shRNA we chose (shSKIL\#4) (see qPCR and western blot results in Fig. 2a and S1E), CALU-3 and NCI-H520 showed significantly decreased viability, colony formation (proliferation), invasion, and migration abilities (Fig. 2b-g). Measurement of EMT markers showed decreased expression of SNAIL1, SLUG, and vimentin, and increased expression of E-cadherin in SKIL-silenced CALU-3 and NCI-H520 (Fig. 2h, i), indicating inhibited EMT in those cells. Expression levels of cancer stem cell markers (CD44, CD133, SOX2, OCT3/4, and NANOG) were also decreased after silencing of SKIL in CALU-3 and NCIH520 (Fig. 2j, k). Overall, silencing of SKIL resulted in inhibited malignant phenotypes (viability, proliferation, invasion, migration, EMT, and cancer stem cell viability) in CALU-3 and NCI-H520. Furthermore, we also conducted in vivo xenograft experiments, and results showed a reduced tumor growth in xenograft generated from SKIL-silenced CALU-3 cells (Fig. 2l).

\section{Silencing on SKIL expression increased T cell infiltration and release of chemokines via activation on the STING pathway}

In addition to malignant phenotypes, we further investigated the influence of SKIL on T cell-related immune escape of tumor cells. A mouse-derived lung cancer cell line (M109) was treated with shSKIL (or control shRNA) and used to generate tumor in immunocompetent BALB/ c mice (synergic model). After 21 days, mice were euthanized and tumor blocks were collected. Flow cytometry showed increased number of total $\mathrm{T}$ cell $\left(\mathrm{CD}^{+}\right.$ $\left.\mathrm{CD} 45^{+}\right)$and cytotoxic $\mathrm{T}$ cell $\left(\mathrm{CD}^{+} \mathrm{CD}^{+} 5^{+} \mathrm{CD} 8^{+}\right)$in the 


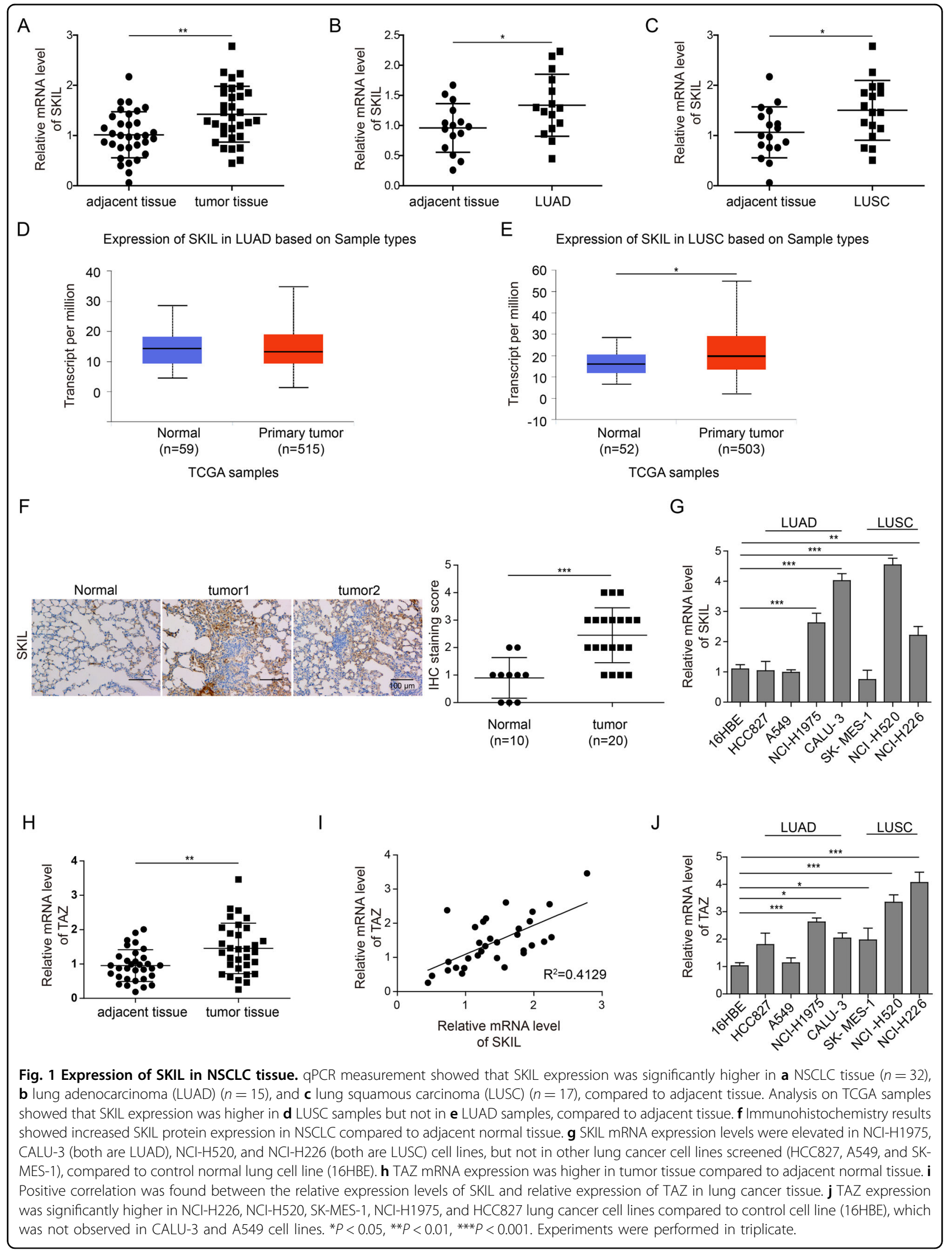




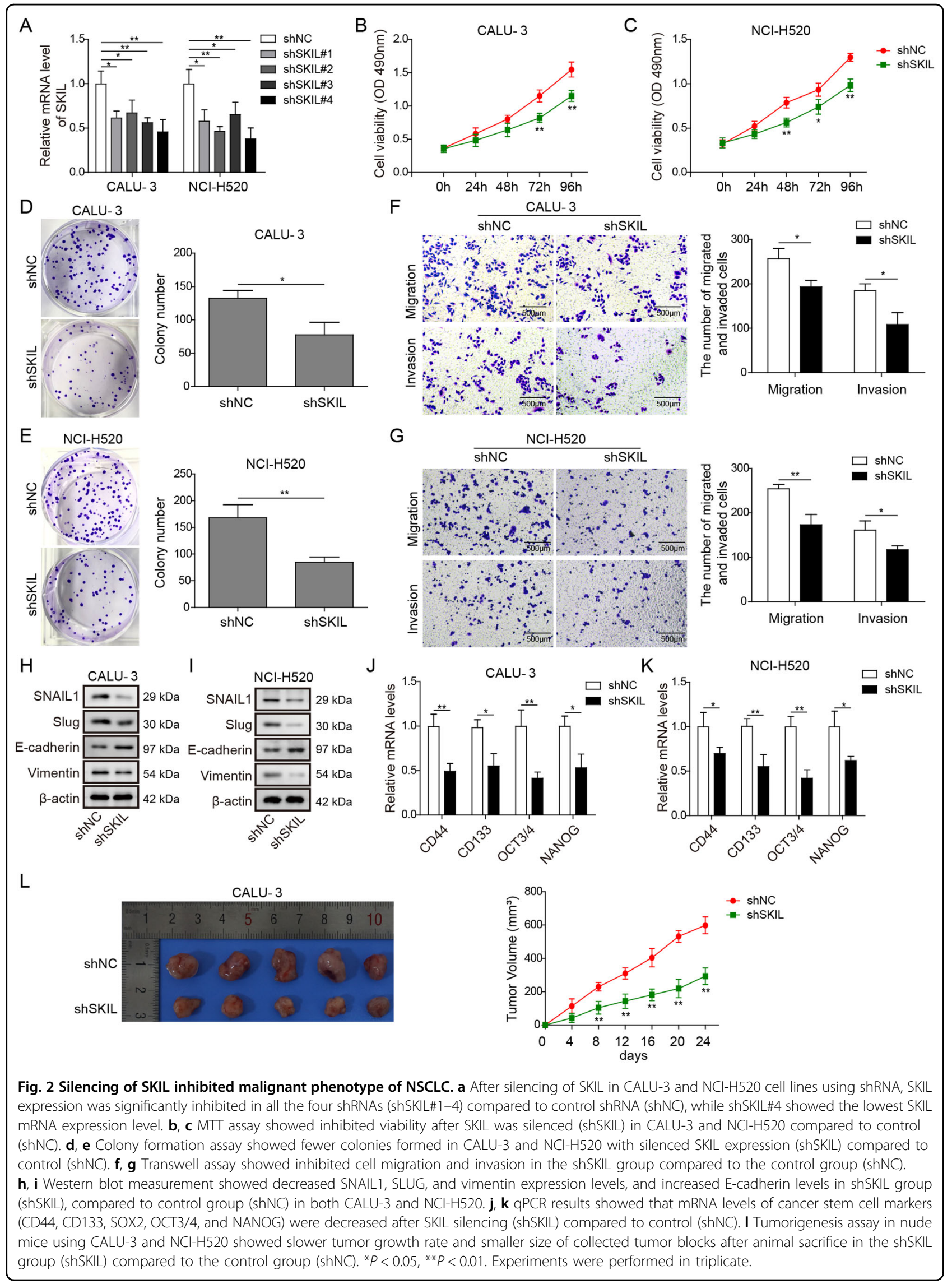




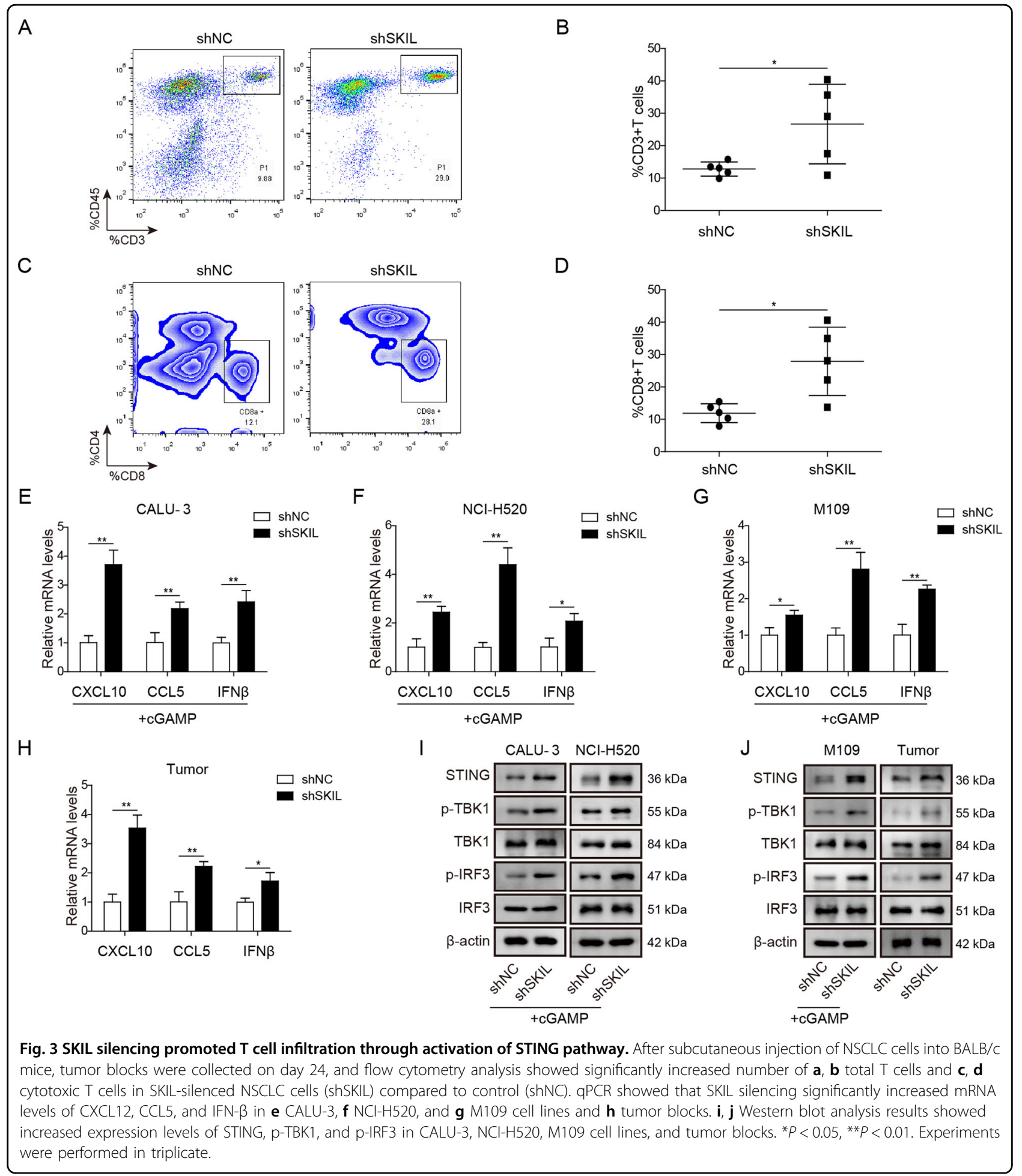

SKIL-silenced xenograft model (Fig. 3a-d) compared to control. qPCR analysis showed that levels of chemokines (CXCL10, CCL5, and IFN- $\beta$ ) which are involved in cytotoxic $\mathrm{T}$ cell recruitment were elevated in SKILsilenced CALU-3 and NCI-H520 at the presence of
STING pathway agonist (cGAMP) and in SKIL-silenced xenograft model (Fig. 3e-h), indicating an activation of IFN- $\beta$ pathway. Expression levels of STING were increased when SKIL was silenced, together with increased phosphorylation of STING downstream 
signaling pathway factors, including TBK1 and transcription factor interferon regulatory factor 3 (IRF3) ${ }^{33}$ in CALU-3 and NCI-H520 at the presence of cGAMP and in SKIL-silenced xenograft model (Fig. 3i, j), indicating that knockdown of SKIL might influence T cell infiltration and release of relevant chemokines through activation of the STING pathway.

\section{Silencing on SKIL expression inhibited autophagy and attenuated TAZ activation}

Since SKIL was reported to regulate autophagy in several types of cancer ${ }^{19,20}$, we also investigated the relationship between SKIL and autophagy in NSCLC. Measurement on autophagy-related markers (LC3-II/I, p62, and Beclin-1) showed decreased levels of those markers in SKIL-silenced CALU-3 and NCI-H520 (Fig. 4a). Formation of autophagosome was also inhibited in SKIL-silenced cell lines, indicated by decrease of cells with LC3-positive vacuoles (Fig. 4b, c). Previous study found that SKIL could promote tumorigenesis of breast cancer through enhancement of TAZ signaling ${ }^{32}$. In addition, TAZ was reported to regulate autophagy in different types of cancer ${ }^{31,32}$. Therefore, we also measured levels of TAZ and its downstream pro-tumorigenesis factors, and results showed decreased levels of TAZ and its downstream factors (CTGF, CYR61) in SKIL-silenced cell lines (Fig. 4d), suggesting that SKIL might regulate autophagy via regulation on the TAZ pathway in NSCLC.

We further investigated the possible interaction between SKIL and TAZ. Results showed that SKIL silencing accelerated TAZ degradation when treated with cycloheximide (Fig. 4e, f). Co-immunoprecipitation (coIP) results showed that SKIL could bind to elements of hippo complex (LATS2 and Salvador, Sav), but not TAZ (Fig. 4g). Overexpression of LATS2, an inhibitor of $\mathrm{TAZ}^{32}$, promoted degradation of TAZ through phosphorylation, while SKIL overexpression inhibited phosphorylation and degradation of TAZ without influencing the level of LATS2 (Fig. 4h). Those results suggest that SKIL could indirectly regulate TAZ activation through interaction with LATS2, which might be involved in the regulation of SKIL on autophagy in NSCLC.

\section{TAZ is involved in the induction of autophagy and malignant phenotype of NSCLC cells by SKIL}

To further demonstrate the role of TAZ in the relationship between SKIL and malignant phenotype and autophagy of NSCLC cell, we used vectors to overexpress SKIL in CALU-3 and NCI-H520 and then silenced TAZ expression in those cells (Fig. 5a). Overexpression of SKIL significantly increased cell viability, and invasion and migration abilities in CALU-3 and NCI-H520, while further silencing of TAZ led to decreased cell viability, and migration and invasion abilities compared to SKIL overexpression group (Fig. 5b-e), indicating that TAZ plays key roles in the regulation of malignant phenotype by SKIL. Furthermore, overexpression of SKIL increased expression of SLUG and vimentin, decreased E-cadherin expression, as well as increased levels of cancer stem cell markers (CD44, SOX2, OCT3/4) and autophagy markers (LC3, p62, Beclin-1), while further TAZ silencing reversed the effect of SKIL on those markers compared to SKIL overexpression group (Fig. $5 \mathrm{f}-\mathrm{i}$ ). All those results indicate key roles of TAZ in the regulation of NSCLC malignant phenotype and autophagy by SKIL.

\section{TAZ is involved in the regulation of T cell infiltration and} release of chemokines by SKIL

After silencing TAZ in M109 cell line, we transplanted those cells into BALB/c mice. Tumor blocks were collected after euthanizing mice using carbon dioxide on day 21. Flow cytometry showed increased numbers of total $\mathrm{T}$ cell and cytotoxic $\mathrm{T}$ cell (Fig. S4A-D) in tumor blocks generated by TAZ-silenced M109 cells. qPCR results showed that levels of chemokines (CXCL10, CCL5, IFN$\beta$ ) were increased after silencing of TAZ in CALU-3, NCIH520, and M109 at the presence of STING pathway agonist cGAMP and in SKIL-silenced NSCLC cell-derived tumor blocks (Fig. S4E-H). Subsequent analysis showed that levels of STING and phosphorylation of its pathway downstream factors ( $\mathrm{p}$-TBK1, $\mathrm{p}$-IRF3) were elevated when TAZ was silenced (Fig. S4I, J). Those effects after TAZ silencing were similar to that of SKIL silencing (Fig. 3a, j), indicating that TAZ may be involved in the regulation of $\mathrm{T}$ cell infiltration and release of chemokines by SKIL through STING pathway.

In order to further convince the role of SKIL/TAZ axis in immune escape, we overexpressed SKIL in M109 cells. In those cells, we further silenced the expression of TAZ. Those cells were transplanted into BALB/c mice and tumor blocks were collected after 21 days. After examination of flow cytometry, compared to control, SKILoverexpressed tumor showed lower numbers of total $\mathrm{T}$ cells and cytotoxic $\mathrm{T}$ cells. Further silencing of TAZ significantly increased the numbers of total and cytotoxic $\mathrm{T}$ cells in tumor blocks, compared to SKILoverexpression group (Fig. 6a-d). In addition, levels of chemokines (CXCL10, CCL5, IFN- $\beta$ ) were lower in SKILoverexpressed tumor compared to control, and further TAZ silencing significantly increased chemokine levels in those SKIL-overexpressed tumor (Fig. 6e). Analysis on the expression levels of STING pathway showed that SKIL overexpression decreased STING levels and phosphorylation of its downstream factors (p-TBK1, p-IRF3), compared to control. Further silencing of TAZ significantly increased the levels of STING, p-TBK1, and pIRF3, compared to SKIL-overexpression group (Fig. 6f). Those findings further demonstrated the important roles 


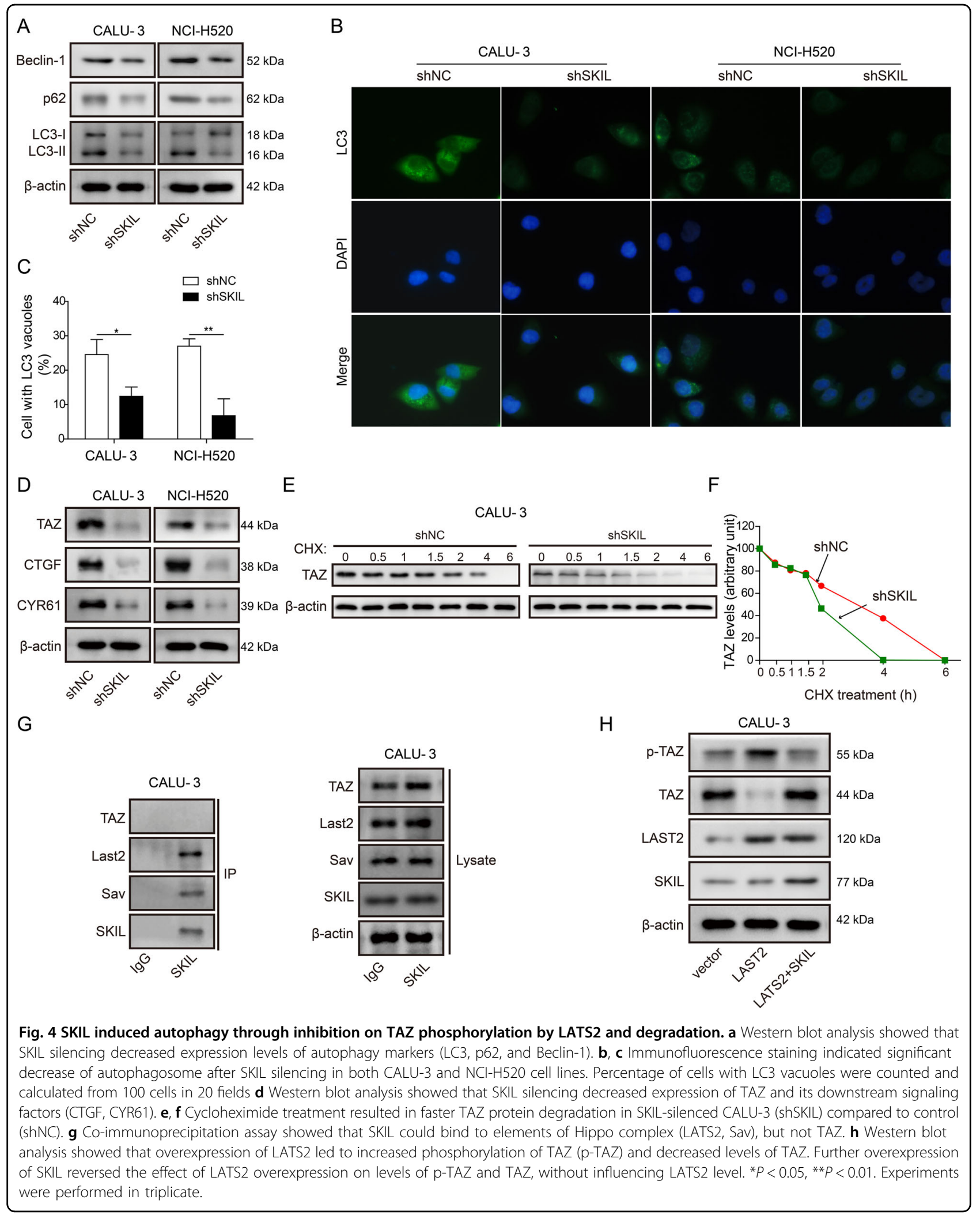




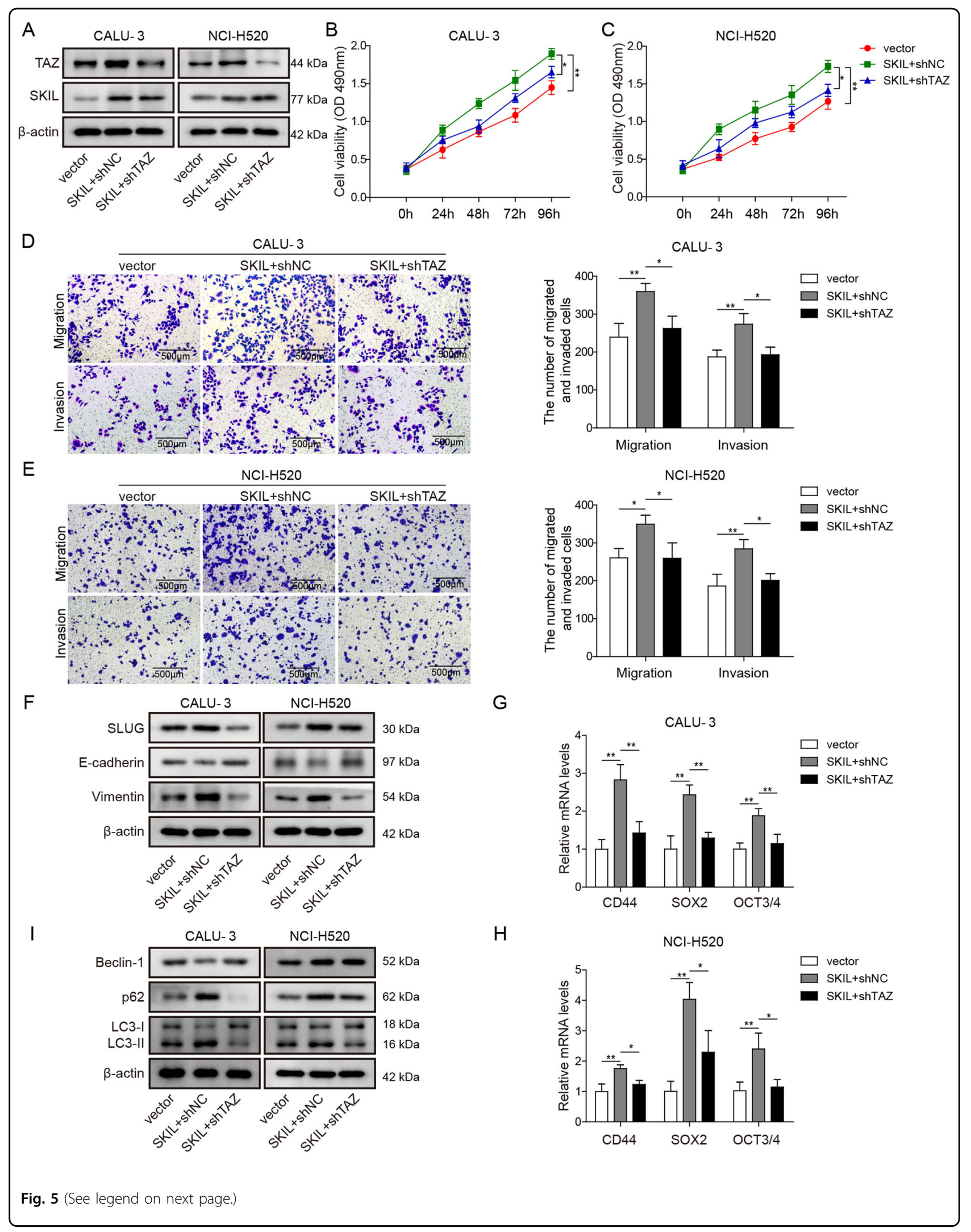


(see figure on previous page)

Fig. 5 TAZ was involved in SKIL-induced autophagy and tumorigenesis. a Western blot analysis showed increased SKIL expression after transfection of vectors carrying SKIL gene (SKIL), and further transfection of shRNA targeting TAZ (shTAZ) decreased TAZ expression. SKIL overexpression in CALU-3 and NCI-H520 (SKIL + shNC) increased b, c cell viability and d, e ability of migration and invasion compared to control (vector), which was reversed by further silencing of TAZ (SKIL + shTAZ) in those cells. f Western blot analysis on EMT-related factors showed that SKIL overexpression increased levels of SLUG and vimentin, and decreased levels of E-cadherin in both CALU-3 and NCI-H520, which was reversed by TAZ silencing. Western blot or qPCR measurement showed that levels of $\mathbf{g}, \mathbf{h}$ cancer stem cell markers (CD44, SOX2, OCT3/4) and $\mathbf{i}$ autophagy-related proteins (LC3, p62, Beclin-1) were increased by SKIL overexpression, which was reversed by further silencing of TAZ in those cells. ${ }^{*} P<0.05$, ${ }^{* *} P<0.01$. Experiments were performed in triplicate.

of SKIL/TAZ axis in immune escape through regulation on STING pathway.

TAZ and autophagy are involved in the regulation of T cell
infiltration and release of chemokines by SKIL Instead of silencing SKIL or TAZ, we also overexpressed the expression of those two factors and used an autophagy inhibitor, bafilomycin, to treat CALU-3 and NCI-H520. Using cGAMP to activate STING pathway, both SKIL and TAZ overexpression resulted in decreased STING levels, which could be canceled by inhibition on autophagy using bafilomycin (Fig. 7a). Similarly, overexpression of SKIL and TAZ inhibited STING pathway activation (decreased levels of p-TBK1, p-IRF3) and expression of chemokines (CXCL10, IFN- $\beta$ ), whereas inhibition on autophagy could cancel the inhibition (Fig. $7 \mathrm{~b}-\mathrm{e}$ ). Those results suggest that autophagy was involved in the regulation on STING pathway and T cell activation by SKIL and TAZ.

\section{Discussion}

Our study found that SKIL expression was increased in NSCLC and was associated with its malignant phenotypes and immune escape from $\mathrm{T}$ cell immunity. Further investigation on the underlying mechanism indicated that the promotion of malignant phenotype was through upregulation of TAZ by interaction with Hippo complex factors (LATS2) and subsequent activation of autophagy. The enhanced autophagy also inhibited STING pathway and activation of $\mathrm{T}$ cells, leading to increased immune escape.

Findings of previous studies indicated complex roles of SKIL in tumorigenesis. Expression of SKIL was elevated in many types of cancer ${ }^{16-18,34}$, but was also found to be inhibited in some subtypes of esophageal, ovarian, pancreatic, and breast cancer ${ }^{22,23}$. Chromosome region $3 q 26$, which contains SKIL gene, was found to be amplified in $31.2 \%$ of NSCLC ${ }^{18}$, and similarly, our results showed that SKIL expression was elevated in tumor samples of NSCLC patients (see Fig. 1). In addition, for the first time, we demonstrated that SKIL could induce malignant phenotype and immune escape in NSCLC (Figs. 2 and 3). Further investigation showed that those effects of SKIL were dependent on upregulation of TAZ expression by interaction between SKIL and Hippo complex elements, Lats2 and Sav (Figs. 4 and 5). This finding was consistent with previous findings that SKIL (SnoN) promoted TAZ signaling through antagonizing Hippo complex in breast cancer $^{32}$. TAZ was found to be over-activated in most types of cancer and is important for tumorigenesis ${ }^{30}$. Through upregulation on downstream factors (amphiregulin, CTGF and CYR61), TAZ could induce many malignant phenotypes of cancer, e.g., cancer stem cell viability, EMT, increased migration and invasion abilities, and higher potential of metastasis ${ }^{30}$. On the other hand, YAP/TAZ was reported to induce autophagy, and this YAP/TAZ-autophagy axis was involved in contact inhibition between normal cells, which was abnormally activated in cancer cells ${ }^{31}$. Our study results showed that SKIL induced autophagy in NSCLC cell lines, and TAZ was essential in this induction (Fig. 5i). Together, those findings indicate that in addition to TAZ signaling pathway (CTGF, CYR61), SKIL may also induce malignant phenotype of NSCLC cells through TAZ-autophagy axis.

Autophagy is a highly conserved cellular mechanism to sequestrate and degrade damaged cytoplasmic structures, which plays key roles in management of cell stress and survival $^{35}$. Defected autophagy is commonly observed in cancer, and is involved in many malignant phenotypes of tumor, including motility, invasion, cancer stem cell viability, EMT, metastasis, etc. ${ }^{35}$. Previous studies also found roles of autophagy in immune response (e.g. affecting release of cytokines, survival, and activation of lymphoid cells), and defects in autophagy could promote immune escape of developing tumors ${ }^{36}$. SOX2 was found to induce escape of HNSC cells from $\mathrm{T}$ cell immunity through autophagy-dependent inhibition on STING pathway ${ }^{37}$. Similarly, our results showed that SKIL induced immune escape of NSCLC cells from $\mathrm{T}$ cell immunity through autophagy-dependent inhibition on STING pathway (Fig. 7 ), and TAZ was involved in the activation of autophagy in this signaling pathway (Fig. S4). Considering the previous findings that TAZ could regulate expression of SOX2 (ref. ${ }^{38}$ ) which could also contribute to the regulation of autophagy and STING pathway $^{37}$, and p62 (another critical autophagy-related protein) could attenuate STING pathway ${ }^{39}$, the SKIL/TAZ-induced inhibition on STING pathway may be mediated by multiple signaling pathways. On the other hand, STING was 


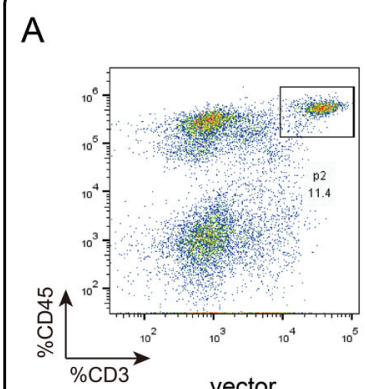

vector

C

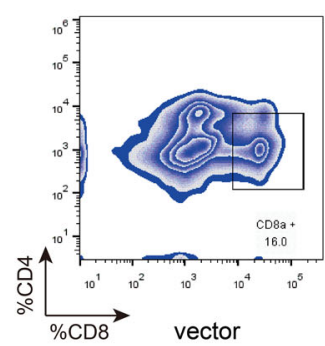

E

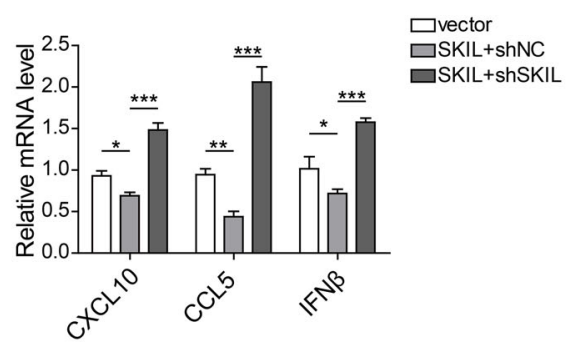

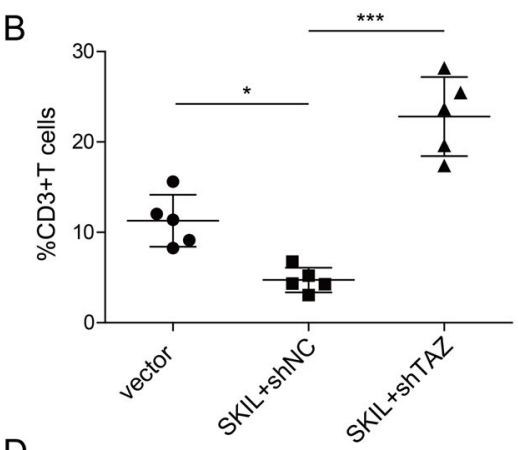

$\mathrm{D}$

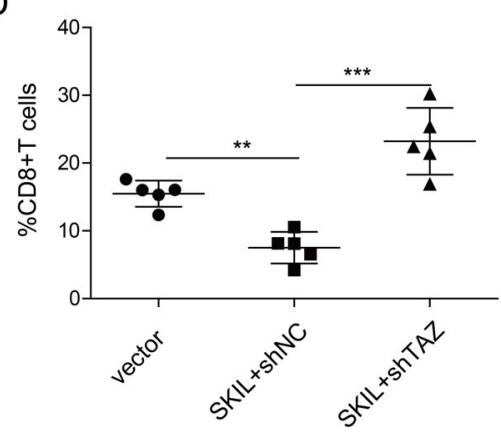

F
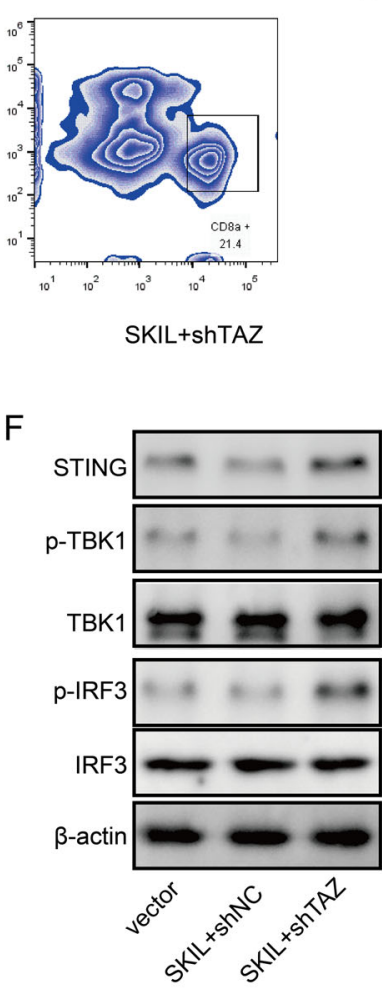

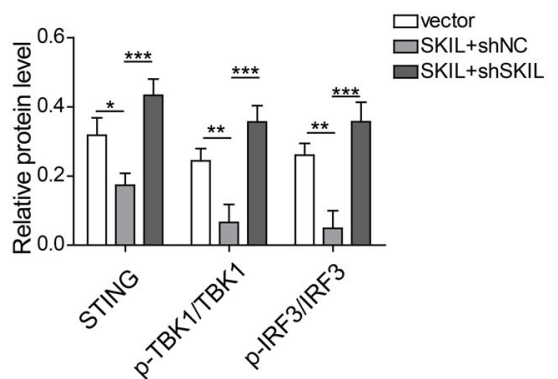

Fig. 6 TAZ silencing promoted T cell infiltration in SKIL-overexpressed tumors through activation of STING pathway. M109 cell line with SKIL overexpression, with both SKIL overexpression and TAZ silencing, or control M109 mice lung cancer cell line were injected subcutaneously into BALB/ c mice. On day 21, mice were euthanized and tumor blocks were collected. a, b Flow cytometry showed lower number of total T cells $\left(C D 45^{+} C D 3^{+}\right)$ in SKIL-overexpressed tumor compared to control group. Tumors with both SKIL overexpression and TAZ silencing showed significantly higher number of total T cells, compared to tumors with SKIL overexpression. c, d Flow cytometry analysis showed decreased cytotoxic T cells $\left(C D 4^{+} \mathrm{CD} 8^{+}\right)$ in tumors with SKIL overexpression, compared to control. Further silencing of TAZ in those cells significantly increased numbers of cytotoxic T cells in those tumors, compared to tumors with SKIL overexpression. e SKIL-overexpressed tumors showed lower levels of CXCL10, CCL5, and IFN- $\beta$ compared to control. Tumors with both SKIL-overexpression and TAZ silencing showed significantly higher levels of CXCL10, CCL5, and IFN- $\beta$, compared to SKIL-overexpression group. $\mathbf{f}$ Expression levels of STING, p-TBK1, and p-IRF3 were decreased in SKIL-overexpressed tumors compared to control tumor. Tumors with both SKIL overexpression and TAZ silencing showed significantly higher STING expression and levels of p-TBK1 and p-IRF3 compared to tumors with SKIL overexpression only. ${ }^{*} P<0.05,{ }^{*} P<0.01$. Experiments were performed in triplicate.

reported to induce autophagy, which is important in clearance of DNA and viruses in cytoplasm $^{40}$. The induction of autophagy by STING was thought to be a primordial function of cGAS pathway, but may also be considered as a feedback mechanism for the regulation of STING pathway by autophagy. More investigations are required to further clarify their relationship.
In summary, our study showed that SKIL expression was increased in NSCLC, and SKIL promoted malignant phenotypes of NSCLC cells via TAZ-dependent upregulation of autophagy. In addition, to the best of our knowledge, our study is the first to show roles of SKIL in immune escape of cancer cells, and to illustrate the underlying mechanisms which involved upregulation of 


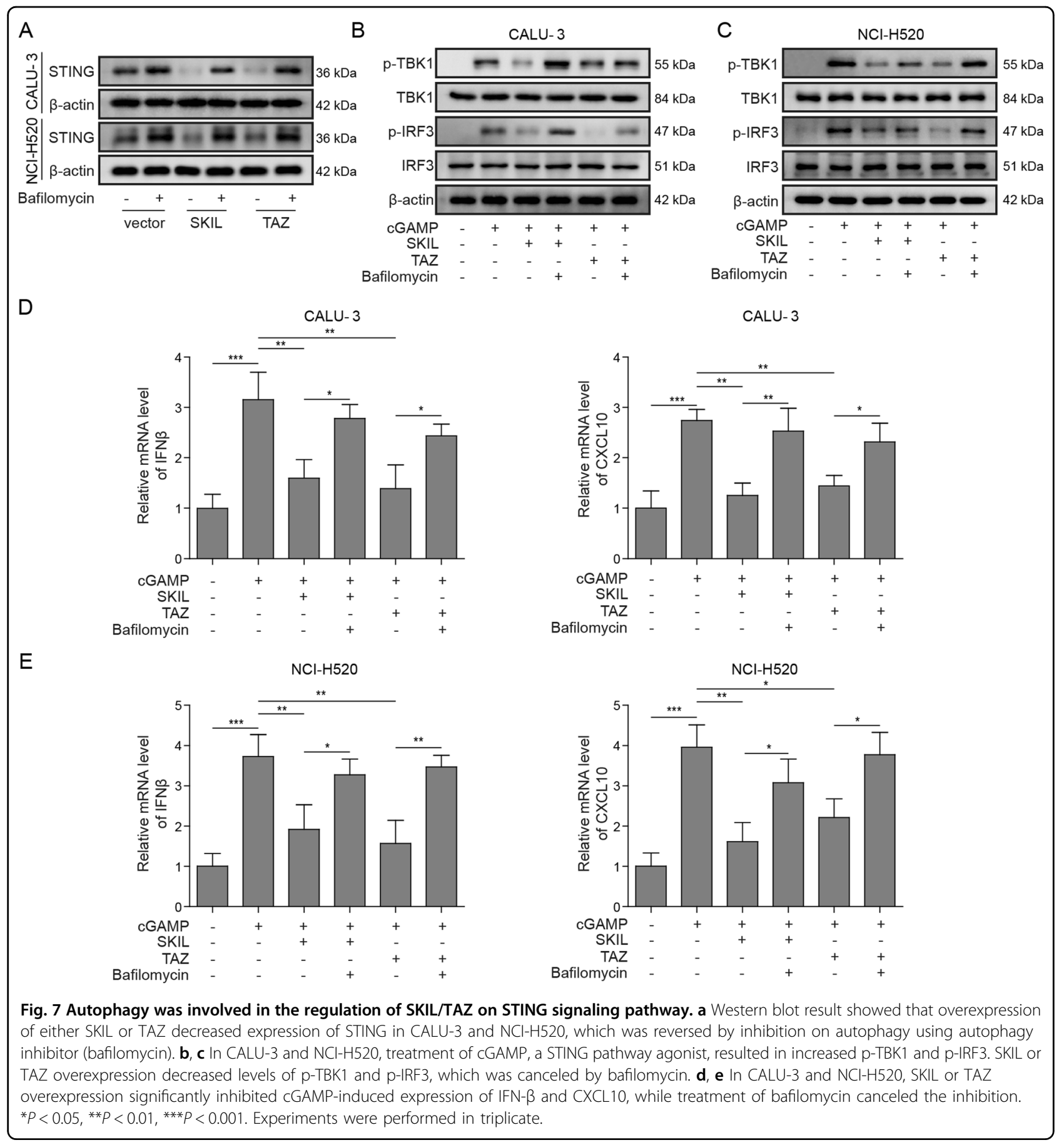

TAZ and autophagy, and subsequent inhibition on STING pathway. Further studies are needed to clarify more details in roles of SKIL in tumorigenesis and underlying molecular mechanisms.

\section{Author contributions}

Guarantor of integrity of the entire study, study design, and manuscript review: X.-L.L.; study concepts, definition of intellectual content, and manuscript editing: F.M.; literature research and data acquisition: M.-G.D.; clinical studies and experimental studies: Y.-Y.L.; data analysis: L.-H.L.; statistical analysis: Y.-H.F.; manuscript preparation: S.J.

\section{Conflict of interest}

The authors declare that they have no conflict of interest.

\section{Ethics approval}

Our study protocol was approved by Institutional Ethics Committee of Central South University. 


\section{Publisher's note}

Springer Nature remains neutral with regard to jurisdictional claims in published maps and institutional affiliations.

Supplementary Information accompanies this paper at (https://doi.org/ 10.1038/s41419-020-03200-7).

Received: 8 April 2020 Revised: 13 October 2020 Accepted: 15 October 2020

Published online: 02 December 2020

\section{References}

1. Torre, L. A., Siegel, R. L., Ward, E. M. \& Jemal, A. Global cancer incidence and mortality rates and trends_-an update. Cancer Epidemiol. Biomark. Prev. 25, 16-27 (2016).

2. Lemjabbar-Alaoui, H., Hassan, O. U., Yang, Y. W. \& Buchanan, P. Lung cancer: biology and treatment options. Biochim. Biophys. Acta 1856, 189-210 (2015).

3. Carbone, D. P., Gandara, D. R., Antonia, S. J., Zielinski, C. \& Paz-Ares, L. Nonsmall-cell lung cancer: role of the immune system and potential for immunotherapy. J. Thorac. Oncol. 10, 974-984 (2015).

4. Reck, M. Pembrolizumab as first-line therapy for metastatic non-small-cell lung cancer. Immunotherapy 10, 93-105 (2018).

5. Deng, L. et al. STING-dependent cytosolic DNA sensing promotes radiationinduced type I interferon-dependent antitumor. Immunity 41, 843-852 (2014).

6. Woo, S. R. et al. STING-dependent cytosolic DNA sensing mediates innate immune recognition of immunogenic tumors. Immunity 41, 830-842 (2014).

7. Sivick, K. E. et al. Magnitude of therapeutic STING activation determines $C D 8\left(^{+}\right.$ T cell-mediated anti-tumor immunity. Cell Rep. 25, 3074-3085.e3075 (2018).

8. Kitajima, S. et al. Suppression of STING associated with LKB1 loss in KRASdriven lung cancer. Cancer Discov. 9, 34-45 (2019).

9. Konno, H. et al. Suppression of STING signaling through epigenetic silencing and missense mutation impedes DNA damage mediated cytokine production. Oncogene 37, 2037-2051 (2018).

10. Khoo L. T. \& Chen L. Y. Role of the CGAS-STING pathway in cancer development and oncotherapeutic approaches. EMBO Rep. 2018, 19.

11. Ishikawa, H., Ma, Z. \& Barber, G. N. STING regulates intracellular DNA-mediated, type I interferon-dependent innate immunity. Nature 461, 788-792 (2009).

12. Sun, L., Wu, J., Du, F., Chen, X. \& Chen, Z. J. Cyclic GMP-AMP synthase is a cytosolic DNA sensor that activates the type I interferon pathway. Science $\mathbf{3 3 9}$ 786-791 (2013).

13. Sen, T. et al. Targeting DNA damage response promotes antitumor immunity through STING-mediated T-cell activation in small cell lung cancer. Cancer Discov. 9, 646-661 (2019).

14. Shen, J. et al. PARPi triggers the STING-dependent immune response and enhances the therapeutic efficacy of immune checkpoint blockade independent of BRCAness. Cancer Res. 79, 311-319 (2019).

15. Deheuninck, J. \& Luo, K. Ski and SnoN, potent negative regulators of TGF-beta signaling. Cell Res. 19, 47-57 (2009).

16. Akagi, l. et al. SnoN overexpression is predictive of poor survival in patients with esophageal squamous cell carcinoma. Ann. Surg. Oncol. 15, 2965-2975 (2008).

17. Zhang, F. et al. Ski-related novel protein N (SnoN), a negative controller of transforming growth factor-beta signaling, is a prognostic marker in estrogen receptor-positive breast carcinomas. Cancer Res. 63, 5005-5010 (2003).
18. Hagerstrand, D. et al. Systematic interrogation of 3q26 identifies TLOC1 and SKIL as cancer drivers. Cancer Discov. 3, 1044-1057 (2013).

19. Raffoul, F., Campla, C. \& Nanjundan, M. SnoN/SkiL, a TGFbeta signaling mediator: a participant in autophagy induced by arsenic trioxide. Autophagy $\mathbf{6}$, 955-957 (2010)

20. Smith, D. M. et al. Arsenic trioxide induces a beclin-1-independent autophagic pathway via modulation of SnoN/SkiL expression in ovarian carcinoma cells. Cell Death Differ. 17, 1867-1881 (2010).

21. Lazarus, K. A. et al. BCL11A interacts with SOX2 to control the expression of epigenetic regulators in lung squamous carcinoma. Nat. Commun. 9, 3327 (2018).

22. Jahchan, N. S., Ouyang, G. \& Luo, K. Expression profiles of SnoN in normal and cancerous human tissues support its tumor suppressor role in human cancer. PLoS ONE 8, e55794 (2013).

23. Shinozuka, E. et al. SnoN/SKIL modulates proliferation through control of hsamiR-720 transcription in esophageal cancer cells. Biochem. Biophys. Res. Commun. 430, 101-106 (2013).

24. Zanconato, F., Battilana, G., Cordenonsi, M. \& Piccolo, S. YAP/TAZ as therapeutic targets in cancer. Curr. Opin. Pharmacol. 29, 26-33 (2016).

25. Piccolo, S., Dupont, S. \& Cordenonsi, M. The biology of YAP/TAZ: hippo signaling and beyond. Physiol. Rev. 94, 1287-1312 (2014).

26. Zanconato, F., Cordenonsi, M. \& Piccolo, S. YAP/TAZ at the roots of cancer Cancer Cell 29, 783-803 (2016).

27. Lamar, J. M. et al. SRC tyrosine kinase activates the YAP/TAZ axis and thereby drives tumor growth and metastasis. J. Biol. Chem. 294, 2302-2317 (2019).

28. Zhao, B., Li, L., Tumaneng, K., Wang, C. Y. \& Guan, K. L. A coordinated phosphorylation by Lats and CK1 regulates YAP stability through SCF(beta-TRCP). Genes Dev. 24, 72-85 (2010).

29. Zhao, B. et al. Inactivation of YAP oncoprotein by the Hippo pathway is involved in cell contact inhibition and tissue growth control. Genes Dev. 21 2747-2761 (2007).

30. Moroishi, T., Hansen, C. G. \& Guan, K. L. The emerging roles of YAP and TAZ in cancer. Nat. Rev. Cancer 15, 73-79 (2015).

31. Pavel, M. et al. Contact inhibition controls cell survival and proliferation via YAP/TAZ-autophagy axis. Nat. Commun. 9, 2961 (2018).

32. Zhu, Q. et al. SnoN antagonizes the Hippo kinase complex to promote TAZ signaling during breast carcinogenesis. Dev. Cell 37, 399-412 (2016).

33. Tanaka, Y. \& Chen, Z. J. STING specifies IRF3 phosphorylation by TBK1 in the cytosolic DNA signaling pathway. Sci. Signal. 5, ra20 (2012).

34. Liu, C., Zhang, H., Zang, X., Wang, C. \& Kong, Y. The influence of SnoN gene silencing by siRNA on the cell proliferation and apoptosis of human pancreatic cancer cells. Diagn. Pathol. 10, 30 (2015).

35. Mowers, E. E., Sharifi, M. N. \& Macleod, K. F. Autophagy in cancer metastasis. Oncogene 36, 1619-1630 (2017).

36. Ma, Y., Galluzzi, L., Zitvogel, L. \& Kroemer, G. Autophagy and cellular immune responses. Immunity 39, 211-227 (2013).

37. Tan, Y. S. et al. Mitigating SOX2-potentiated Immune escape of head and neck squamous cell carcinoma with a STING-inducing nanosatellite vaccine. Clin. Cancer Res. 24, 4242-4255 (2018).

38. Xiao, H., Jiang, N., Zhou, B., Liu, Q. \& Du, C. TAZ regulates cell proliferation and epithelial-mesenchymal transition of human hepatocellular carcinoma. Cancer Sci. 106, 151-159 (2015)

39. Prabakaran, T. et al. Attenuation of CGAS-STING signaling is mediated by a p62/SQSTM1-dependent autophagy pathway activated by TBK1. EMBO J. 37 (8), e97858 (2018)

40. Gui, X. et al. Autophagy induction via STING trafficking is a primordial function of the cGAS pathway. Nature 567, 262-266 (2019). 